\title{
The Pierre Auger Observatory: latest results and future perspec- tives
}

\author{
Mario Buscemi ${ }^{1,2, a}$ on behalf of the Pierre Auger Collaboration ${ }^{3}$ \\ ${ }^{1}$ Università degli Studi di Catania, Italy \\ ${ }^{2}$ INFN Sezione di Catania, Italy \\ ${ }^{3}$ Observatorio Pierre Auger, Av. San Martín Norte 304, 5613 Malargüe, Argentina \\ Full author list: http://www.auger.org/archive/authors_2017_08.html
}

\begin{abstract}
The Pierre Auger Observatory is the largest ultrahigh-energy cosmic ray observatory in the world. The huge amount of high quality data collected since 2004 up to now led to great improvements in our knowledge of the ultra-energetic cosmic rays. The suppression of the cosmic-ray flux at highest energies was clearly established, and the extra-galactic origin of these particles was confirmed. On the other hand, measurements of the depth of shower maximum indicate a puzzling trend in the mass composition of cosmic rays at energy around the ankle up to the highest energy. The just started upgrade of the Observatory, dubbed AugerPrime, will improve the identification of the mass of primaries allowing us to disentangle models of origin and propagation of cosmic rays.
\end{abstract}

\section{Introduction}

The Pierre Auger Observatory is the largest Observatory for the study of cosmic ray in the world. It is located near Malargüe in the Province of Mendoza, Argentina, and it has been in operation since 2004 [1].

When ultrahigh-energy cosmic rays (UHECRs) enter the Earth's atmosphere they can produce showers of secondary particles that propagate up to reaching the ground. The distribution of particles (electrons, positrons, muons and photons) at ground level strongly depends on the energy and mass of the primary particle that generated the shower. Furthermore, interactions of the secondary particles of a shower with the atmosphere can lead to the excitacion of nitrogen molecules, and then de-excitation via fluorescence light emission. The amount of produced fluorescence light is proportional to the energy that the shower dissipates in the atmosphere, so its detection can provide a calorimetric measurement of the energy of the primary cosmic ray. Moreover measurements of the atmospheric depth at which the number of particle of a shower is the maximum, the $\mathrm{X}_{\max }$ of a shower, is a way to estimate the mass of the primary particle.

One of the main feature of the Observatory is its hydrid design that allows us to exploit both the techniques, indeed a Surface Detector (SD) and a Fluorescence Detector (FD) observe simultaneously UHECRs from a fraction of an $\mathrm{EeV}\left(10^{18} \mathrm{eV}\right)$ to the highest energies ever observed (hundreds of $\mathrm{EeV})$.

\footnotetext{
a e-mail: mario.buscemi@ct.infn.it
} 


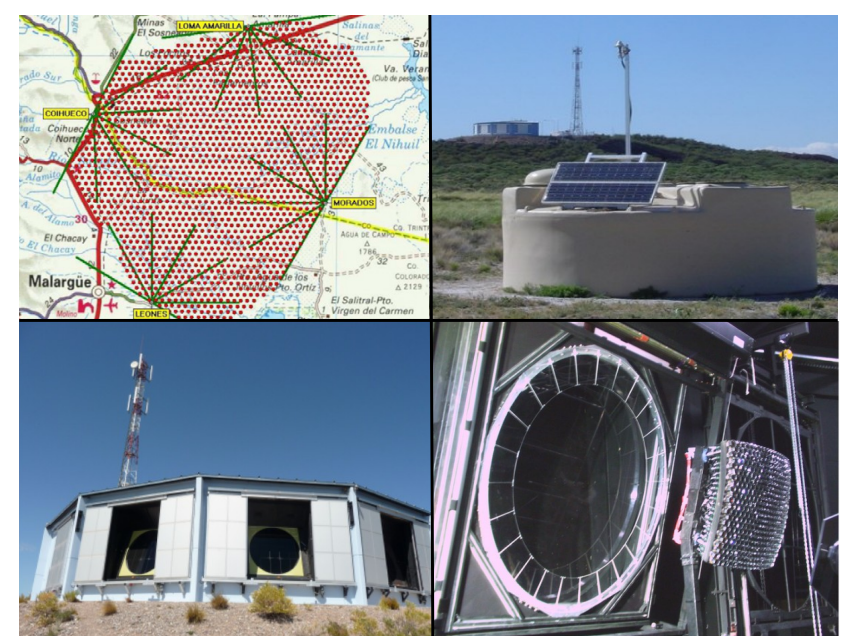

Figure 1. Top-Left: map of the Pierre Auger Observatory, each red point represents one WCD, green lines represent the field of view of the fluorescence detector buildings. Top-Right: one of the 1660 WCDs that compone the surface detector. Bottom-Left: a building of the fluorescence detector. Bottom-Right: a camera of the FD containing an array of $22 \times 24$ photomultipliers.

The SD is made of 1600 Water Cherenkov Detectors (WCDs) arranged in a $1500 \mathrm{~m}$ spaced triangular array, covering an area of $\sim 3000 \mathrm{~km}^{2}$, plus a $750 \mathrm{~m}$ spaced nested array of 61 additional WCDs covering an area of $23.5 \mathrm{~km}^{2}$. The FD consists in 27 air fluorescence telescopes placed in four sites around the SD: each telescope collects the fluorescence light originated from a portion of the atmoshere above the SD. 24 telescopes have a field of view of $30^{\circ} \times 30^{\circ}$ in azimuth and elevation, with a minimum elevation of $1.51^{\circ}$ above the horizon (see Figure 1). Three other telescopes, the High Elevation Auger Telescopes (HEAT) point the sky with higher elevation, up to $60^{\circ}$, and are useful to detect less energetic showers in combination with the nested array.

The SD and the FD operate together in a complementary way. The SD is characterized by an 100\% duty cycle, but it allows just indirect measurements of the energy of primary particles (see section 2) and, to date, its measurements are not much sensitive to the chemical composition of cosmic rays. On the other hand, the FD can operate only during moonless clear night, and this reduces its duty cycle to about $15 \%$, but, thanks to the reconstruction of the longitudinal development of showers, it offers the opportunity to estimate the mass composition from the $\mathrm{X}_{\max }$ observable, and to measure the energy released by a shower in a direct, almost calorimetric way. Events simultaneously recorded by the FD and the SD, the so-called hybrid events, make possible the energy cross calibration between the two detectors.

\section{The energy spectrum}

The flux of cosmic rays reaching the Earth varies with their energy, and the shape of the spectrum at the highest energies is linked to the origin and nature of UHECRs. The energy spectrum measured at the Pierre Auger Observatory is obtained using an exposure exceeding $67000 \mathrm{~km}^{2} \mathrm{sr}$ yr cumulated since January 2004 until December 2016 [2]. The spectrum, shown in Figure 2-Left, results from the combination of four different data samples: events collected by the $1500 \mathrm{~m}$ spaced array having an 

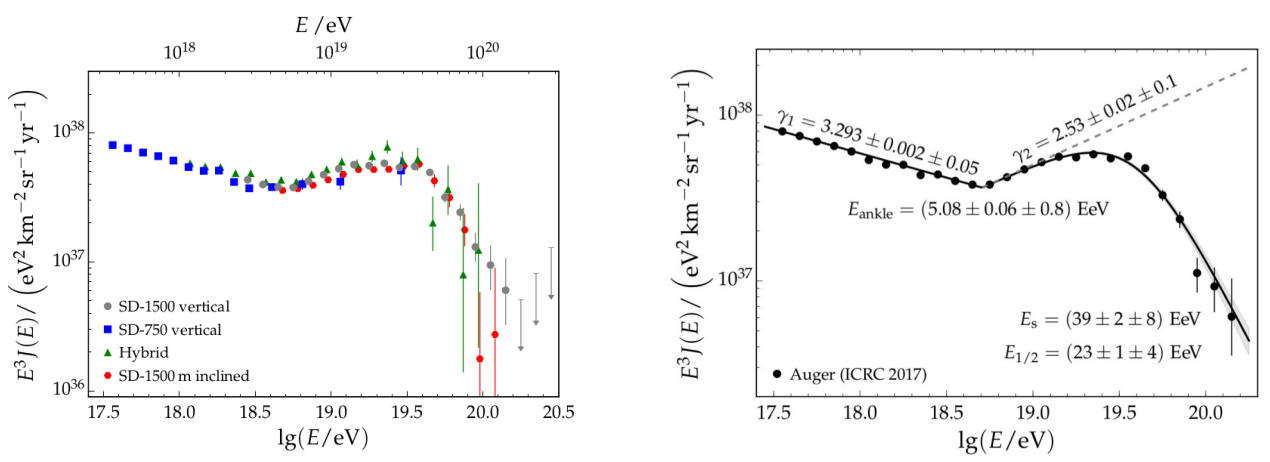

Figure 2. Left: The energy spectra obtained with SD 1500 vertical and inclined, hybrid and SD 750 events. Right: The combined spectrum and the fitting function with the fitting parameters.

incident zenith angle $\theta<60^{\circ}$ (SD-1500 vertical) or zenith angle $60^{\circ}<\theta<80^{\circ}$ (SD-1500 inclined), data from the $750 \mathrm{~m}$ spaced array (SD-750 vertical), and hybrid events from the FD. The energy estimator of the vertical SD events is based on the lateral distribution of secondary particles on ground at an optimal distance from the shower core. For the SD-1500, the optimal distance is 1000 $\mathrm{m}$, for the SD-750 is $450 \mathrm{~m}$. The energy estimator decreases with zenith angle for a given energy due to the attenuation of the shower when crossing the atmosphere: this dependence can be removed by using the Constant Intensity Cut method [3] converting the estimator S(1000) to the equivalent signal at median zenith angle of $38^{\circ}\left(\mathrm{S}_{38}\right)$. The energy scale is provided by the correlation beetwen the estimator $S_{38}$ and the calorimetric energy measured by the fluorescence detector. It spans from $10^{17.5}$ $\mathrm{eV}$ up to the highest energies and its systematic uncertantiy is $\sim 14 \%$. The systematic uncertainties on the flux are between $5 \%$ and $10 \%$.

A maximum likelihood fit on all the four data sets returns the combined spectrum that is shown in Figure 2-Right. The flux normalizations are used as additional constraints to obtain the flux scaling factors that match them: $(-0.8 \pm 0.2) \%$ for the SD 1500 vertical, $(1 \pm 4) \%$ for the SD 750, $(5.4 \pm$ $0.7) \%$ for the SD 1500 horizontal and $(-6 \pm 2) \%$ for the hybrid. The combined spectrum is fitted by the function:

$$
J_{\text {unf }}(E)= \begin{cases}J_{0}\left(\frac{E}{E_{\text {ankle }}}\right)^{-\gamma_{1}} & E<E_{\text {ankle }} \\ J_{0}\left(\frac{E}{E_{\text {ankle }}}\right)^{-\gamma_{2}}\left[1+\left(\frac{E_{\text {ankle }}}{E_{s}}\right)^{\Delta \gamma}\right]\left[1+\left(\frac{E}{E_{S}}\right)^{\Delta \gamma}\right]^{-1} & E>E_{\text {ankle }}\end{cases}
$$

The spectrum is characterized by an ankle at $\mathrm{E}_{\text {ankle }}=(5.08 \pm 0.06$ (stat.) \pm 0.8 (syst. $\left.)\right) \times 10^{18} \mathrm{eV}$. The suppression is at $\mathrm{E}_{s}=(3.9 \pm 0.2$ (stat.) \pm 0.8 (syst. $\left.)\right) \times 10^{19} \mathrm{eV}$. The energy $\mathrm{E}_{1 / 2}$ at which the integral spectrum drops by a factor of two below what would be the expected with no steepening is $\mathrm{E}_{1 / 2}=$ $(2.26 \pm 0.08$ (stat.) \pm 0.4 (syst.) $) \times 10^{19} \mathrm{eV}$. The spectral indexes are: $\gamma_{1}=3.293 \pm 0.002$ (stat.) \pm 0.05 (syst.), $\gamma_{2}=2.53 \pm 0.02$ (stat.) \pm 0.1 (syst.) while $\Delta \gamma=2.5 \pm 0.1$ (stat.) \pm 0.4 (syst.). 

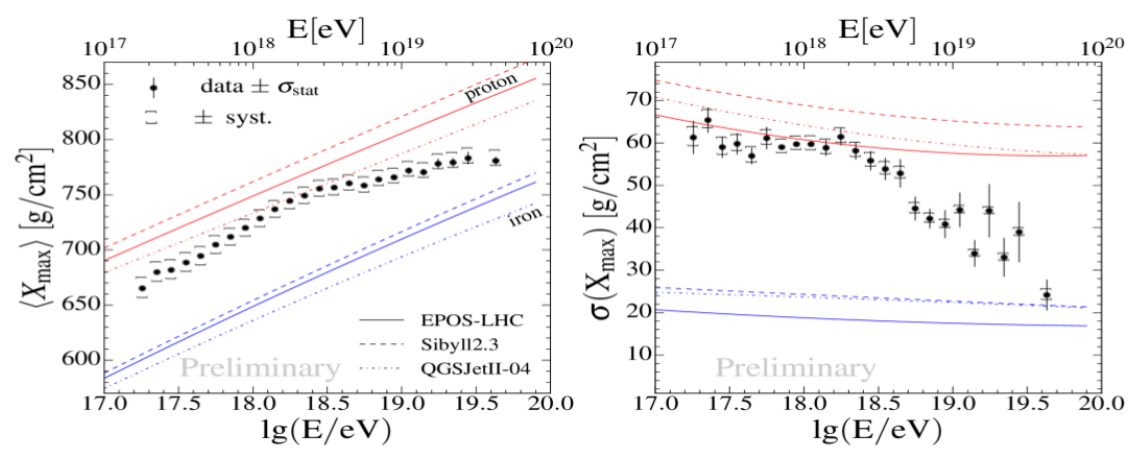

Figure 3. Left: mean value of the $X_{\max }$ distribution as a function of the energy. Right: fluctuations of $X_{\max }$ as a function of the energy [6]. Measurements indicate that the mean primary mass is becoming lighter with increasing energy between $10^{17.2} \mathrm{eV}$ and $10^{18.33} \mathrm{eV}$ and heavier at energies above $10^{18.33} \mathrm{eV}$

\section{The mass composition}

The identification of the acceleration mechanism and the understanding of the shape of the spectrum are some of the less clear puzzles related to UHECRs. The knowledge of the composition of cosmic rays is a keystone to clarify these enigma. The most direct observable to infer the composition from air-shower measurements is the $\mathrm{X}_{\max }$ that at the Pierre Auger Observatory is measured using the fluorescence detector including HEAT. Latest measurements, reported in [4], contain more than five years of calibrated HEAT data, from 1 June 2010 to 31 December 2015, extending the previous measurement of the $\mathrm{X}_{\max }$ distributions [5] from $10^{17.8} \mathrm{eV}$ down to $10^{17.2} \mathrm{eV}$.

The mean value of the $\mathrm{X}_{\max }$ distribution as a function of the energy is shown in Figure 3. The observed elongation rate, defined as the rate of change of $\left\langle X_{\max }>\right.$ is $(80 \pm 1) \mathrm{gcm}^{-2}$ per energy decade between $10^{17.2} \mathrm{eV}$ and $10^{18.33} \mathrm{eV}$. The elongation rate value expected for a constant mass composition is about $60 \mathrm{gcm}^{-2}$ per decade, so measurements indicates that in this energy range the mean primary mass is becoming lighter with increasing energy. On the contrary at energies above $10^{18.33} \mathrm{eV}$ the elongation rate becomes significantly smaller, $(26 \pm 2) \mathrm{gcm}^{-2}$ per decade, suggesting that the composition is becoming heavier with increasing energy. The same considerations arise from the fluctuations of $\mathrm{X}_{\max }$ in Figure 3-Right: their decreasing at energy above $10^{18.3} \mathrm{eV}$ provides an indication that the composition is becoming heavier with increasing energy.

A detailed study of the $\mathrm{X}_{\max }$ distributions was performed at different energies. A result is that at energy above $5 \times 10^{18} \mathrm{eV}$ the distribution can be fitted only when intermediate masses are included in the chemical composition. This is a strong indication that the mass composition of UHECRs does not consist of a pure element composition, but rather a mixture including heavy nuclei with $\mathrm{A}>4$ at the highest energies [6]. 

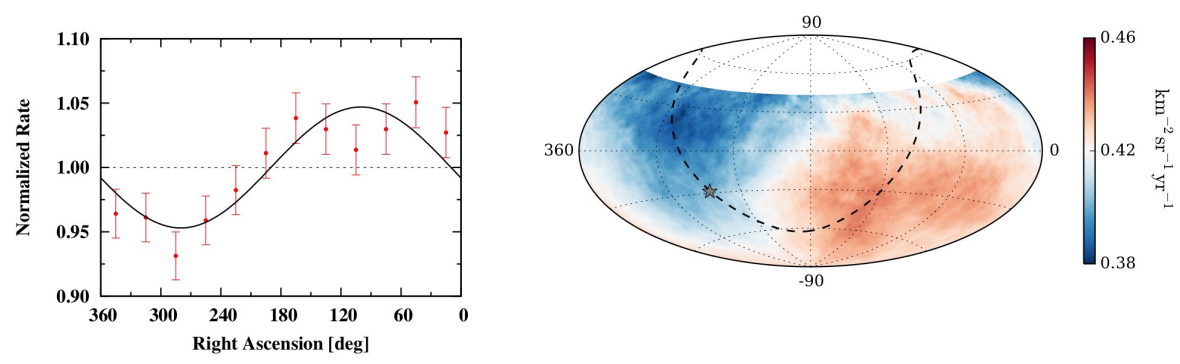

Figure 4. Left: Distribution of the normalized rate of events with $\mathrm{E} \geq 8 \mathrm{EeV}$ as a function of the right ascension. The function corresponding to the first harmonic is also indicated with a solid line, showing that the distribution is compatible with a dipolar modulation. Right: Flux of cosmic rays with $\mathrm{E} \geq 8 \mathrm{EeV}$ smoothed in angular windows of $45^{\circ}$

\section{Arrival directions of UHECRs}

Studies of the distribution of the arrival directions of UHECRs can clarify the origin of these particles, because it is connected to their sources distribution and also to their propagation mechanism. Indications of an anisotropic distributions of sources were found both at large angular scale and at intermediate angular scale.

The distribution at large angular scales of the arrival directions of cosmic rays is studied performing a classical harmonic analysis generalized by introducing weights that take into account possible variations in the coverage of the array arising from the variations in its operating size as a function of time and for the effects of a net tilt of the array surface. The observation of a large-scale anisotropy in arrival directions of cosmic rays above $8 \mathrm{EeV}$ was recently claimed with a significance at a level of more than $5.2 \sigma$ [7]. The observed arrival directions distribution is indicative of an extragalactic origin of UHECRs. Models proposing Galactic sources up to the highest energies require a predominantly heavy composition at $\mathrm{EeV}$ energies which is in disagreement with observations. Another indication of the extragalactic origin of the most energetic cosmic rays is that the maximum of the dipole lies $125^{\circ}$ off the direction of the Galactic center. The distribution of the normalized rate of events with $\mathrm{E} \geq 8 \mathrm{EeV}$ as a function of the right ascension is shown in Figure 4-Left. The solid line indicates the function corresponding to the first harmonic, showing that the distribution is compatible with a dipolar modulation. The flux of the same sample of events smoothed in angular windows of $45^{\circ}$ is shown in Figure 4-Right.

New studies have been performed to correlate anisotropies in intermediate angular scale arrival direction with the directions of candidate UHECRs accelerators. The two largest departures from isotropy above $40 \mathrm{EeV}$, with significance of about $\sim 3 \sigma$, are found around the direction towards Centaurus A and the most luminous AGNs of the Swift-BAT catalog [8]. Furthermore, the gamma rays sources observations of the Fermi -LAT satellite motivated us to examine the correlation of our highest energy events with two nearby populations of extragalactic gamma rays sources, namely star-forming galaxies and AGNs. A $2.7 \sigma$ excess has been found in the directions of the active galaxies, while with the starburst galaxies there is a $4 \sigma$ deviation from isotropy at an intermediate angular scale. 

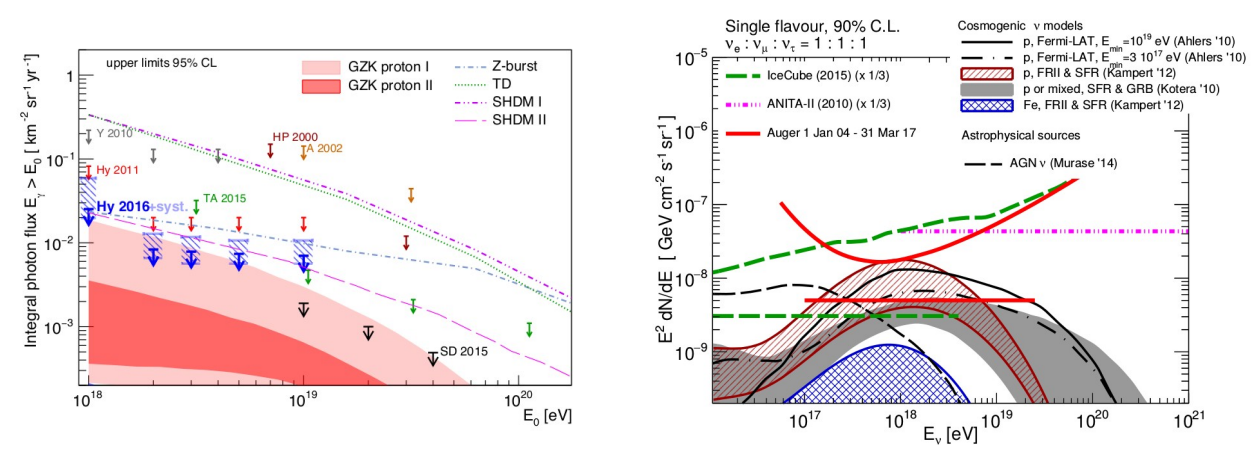

Figure 5. Left: Upper limits on the integral photon flux [9]. The shaded regions and the lines give the predictions for photon fluxes from several production models. Right: Integral and differential upper limit (at 90\% C.L.) for a diffuse neutrino flux [10]. Limits are quoted for a single flavor assuming equal flavor ratios and are displayed along with predictions for several neutrino models.

\section{Search for cosmogenic photons and neutrinos}

Ultra-energetic photons are tracers of the Greisen-Zatsepin-Kuzmin (GZK) process. In these interactions, neutral pions are produced, which subsequently decay into pairs of ultra-energetic photons. If these predicted photons were observed, it would be an indicator for the GZK process being the reason for the observed suppression in the energy spectrum of UHECRs. On average, air showers initiated by ultra-energetic photons differ from air showers generated by hadrons. Photon-induced showers develop deeper in the atmosphere due to the typically smaller multiplicity of electromagnetic interactions, produce a lower average number of muons, and, having a steeper lateral distribution function, are characterized by a smaller footprint on ground. The latest analysis performed by the Pierre Auger Collaboration is applied to hybrid data collected between January 2005 and December 2013 and is described in detail in [9]. Selection criteria are applied to ensure a good geometry and profile reconstruction and a reliable determination of the discriminating observables. After the event selection, 8178 events with energies above $10^{18} \mathrm{eV}$ remained for the analysis. Three events pass the photon candidate cut, a number that is compatible with the expected background for a pure-proton or mixed composition (11.4 and 3.3 respectively). Accordingly to this, upper limits on the integral photon flux at $95 \%$ confidence level (C.L.) are derived (see Figure 5-Left). The current upper limits impose tight constraints on current top-down scenarios proposed to explain the origin of UHECRs.

Neutrinos with an energy of about $10^{18} \mathrm{eV}$ are expected from interactions of UHECRs in the sources or during propagation through the Universe. Since neutrinos are chargeless particles they are not deflected by galactic and extra-galactic magnetic fields and then they are the best probe to point an UHECRs production source. The Pierre Auger Observatory is effective at detecting down-going $\mathrm{EeV}$ neutrino interactions in the atmosphere and Earth-skimming tau neutrinos. Air showers produced by inclined neutrinos that develop deep in the atmosphere can be distinguished from showers due to cosmic rays that interact in the upper layers. Deep neutrino showers produce signals with a time spread broader than that measured in hadron-induced showers, indeed in these the electromagnetic component gets absorbed and only the muon component reaches ground level. Then the energy is deposited in a short time because muons travel nearly parallel to the shower axis and undergo few 

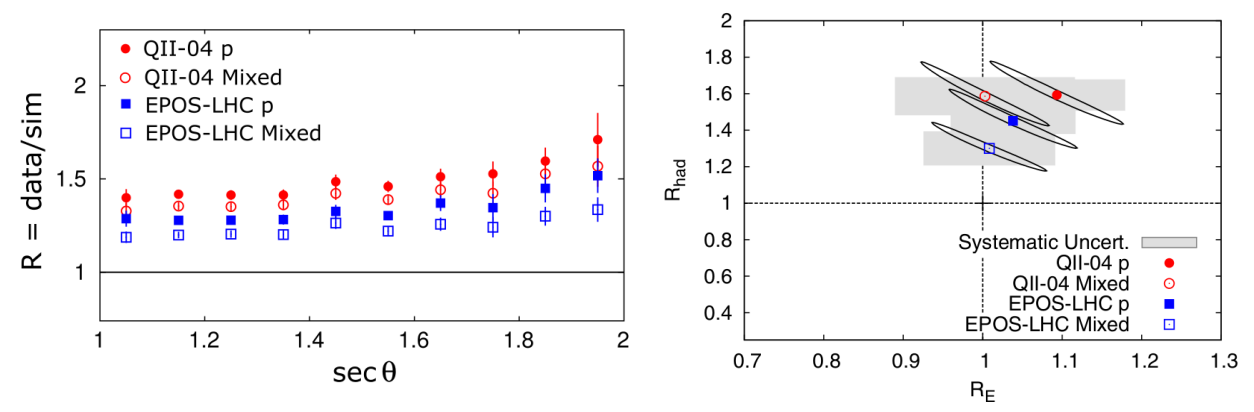

Figure 6. Left: Ratio of $S(1000)$ measured to that predicted for simulated events with matching zenith angle, $\mathrm{X}_{\max }$ and calorimetric FD energy, for QGSJet-II-04 and EPOS-LHC and different composition scenario. Right: Best-fit values of $R_{E}$ and $R_{\text {had }}$ for QGSJet-II-04 and EPOS-LHC, for pure proton (solid circle, square) and mixed composition (open circle, square). The ellipses and gray boxes show the $1 \sigma$ statistical and systematic uncertainties.

interactions. The data set that has been used for neutrino searches spans the period from 1 Jan 2004 to 31 March 2017. No neutrino candidates have been found [10]. The differential and integrated (assuming an $E^{-2}$ spectrum) limits for a diffuse isotropic flux of single flavor neutrinos are shown in Figure 5-Right. There they are compared to those from current neutrino telescopes in the equal flavor scenario and with predictions for several cosmogenic and astrophysical models. Different models for cosmogenic neutrinos that attempt to explain the origin of cosmic rays are excluded at the $90 \%$ C.L., particularly those that assume proton as primaries. Some astrophysical models are also excluded at 90\% C.L. for instance that assuming cosmic ray acceleration in radio-loud AGN.

\section{Hadronic phisycs}

The Pierre Auger Collaboration has already found that the muon number in highly inclined events differs that predicted using the two leading LHC-tuned hadronic event generators for air showers, QGSJet-II-04 and EPOS-LHC [11], but the significance of the inferred muon excess is limited due to the uncertainty in the absolute energy calibration. A new analysis method [12] was recently developed to test hadronic interaction models at the highest energies without relying on the absolute energy calibration. The analysis was applied to 411 vertical hybrid events with $10^{18.8}<\mathrm{E}<10^{19.2} \mathrm{eV}$ recorded between 1 January 2004 and 31 December 2012. Figure 6-Left shows the ratio of S(1000), the ground signal size at $1000 \mathrm{~m}$ from the shower core, for the events in our sample relative to that predicted for simulated events with matching zenith angle, $\mathrm{X}_{\max }$, and calorimetric FD energy, for QGSJet-II-04 and EPOS-LHC. The analysis is done using the mixed composition which reproduces the observed $\mathrm{X}_{\max }$ distribution. The discrepancy between a measured and simulated $\mathrm{S}(1000)$ is evident at all zenith angles and for both models. Simulated $\mathrm{S}(1000)$ signals for given shower $i$, with assumed primary mass $j$, are rescaled to quantify the discrepancy accordig to the equation:

$$
S_{r e s c}\left(R_{E}, R_{h a d}\right)_{i, j}=R_{E} S_{E M, i, j}+R_{h a d} R_{E}^{\alpha} S_{h a d, i, j}
$$

where $S_{E M}$ and $S_{\text {had }}$ are the electromagnetic and the hadronic signal, $R_{E}$ is a rescaling parameter to allow for a possible shift in the FD energy calibration, and $R_{\text {had }}$ is a multiplicative rescaling parameters 

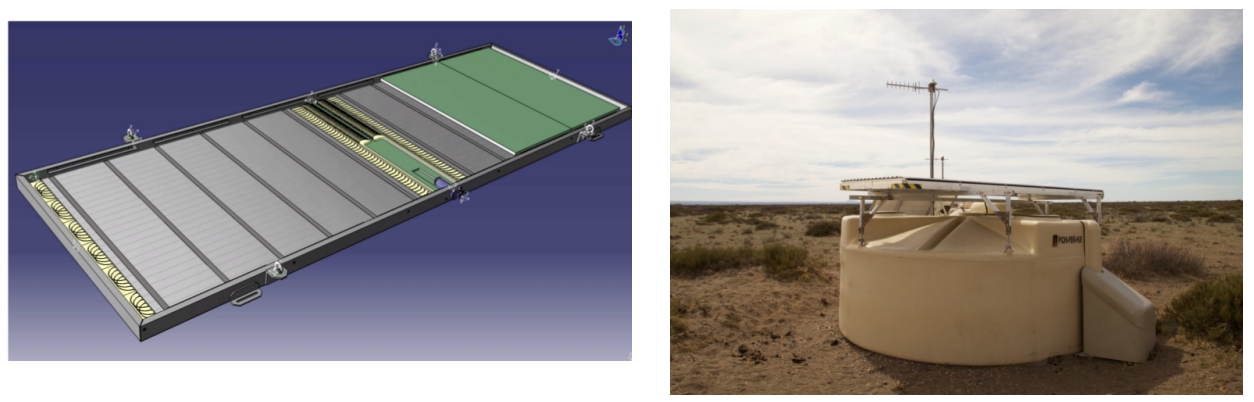

Figure 7. Left: the layout of the Surface Scintillator Detector (SSD). Right: One station of the AugerPrime Engineering Array.

of the hadronic component of the shower. The factor $R_{E}^{\alpha}$ reflects the fact that the hadronic signal increases slower than linearly with energy.

The values of $R_{E}$ and $R_{h a d}$ that maximize the likelihood of the observed ground signals, for the various combinations of simulation models and compositions, are shown in Figure 6-Right. The value of $R_{E}$ is $1.00 \pm 0.10$ for the mixed composition fit with EPOS-LHC and $1.00 \pm 0.14$ for QGSJet II-04, so no energy rescaling is needed.The value of $R_{\text {had }}$ is $1.33 \pm 0.16$ for EPOS-LHC and $1.61 \pm 0.21$ for QGSJet II-04. It confirms that the observed hadronic signal in these UHECR air showers is significantly larger than predicted by models.

\section{The upgrade of the Observatory: AugerPrime}

Results of the Pierre Auger Observatory contributed to improve our understanding of UHECRs, but many issues are still debated. A better knowledge of the chemical composition of UHECRs can help to answer many open questions, such us the origin of the suppression of the flux at highest energies, and can clarify if the measurement of an excess of muons in air showers is due to a new mechanism in the hadronic interactions. Moreover if a significative contribution of protons to the flux was found it could pave the way to the proton-astronomy.

To improve the composition sensitivity of the Pierre Auger Observatory into the flux suppression region, the Auger Collaboration started an upgrade, called AugerPrime, that will extend the data taking of the Observatory up to 2024 [13]. The aim of AugerPrime is to enhance the capability of the Surface Detector to identify the mass of the primary particle on a shower-by-shower basis. AugerPrime consists on many improvements of the Observatory, and in particular the upgrade of the existing WCDs and the addition of a scintillator detector above each of these.

The WCDs will be equipped with a new data acquisition electronics that will increase the data quality thanks to a better timing accuracy and a faster ADC sampling. In addition, the dynamic range of the WCD will be enhanced by a factor 32 with an additional small $(1$ inch) photomultiplier that will be inserted in the WCD [14]. It will reduce the number of saturated events expecially in proximity of the shower axis, where the number of particles of the shower is larger.

A thin scintillation detector, Scintillator Surface Detector (SSD), will be mounted above each WCD, providing a robust and well-understood scheme for particle detection that is sufficiently complementary to the water-Cherenkov technique and permits a good measurement of the density of muons. 

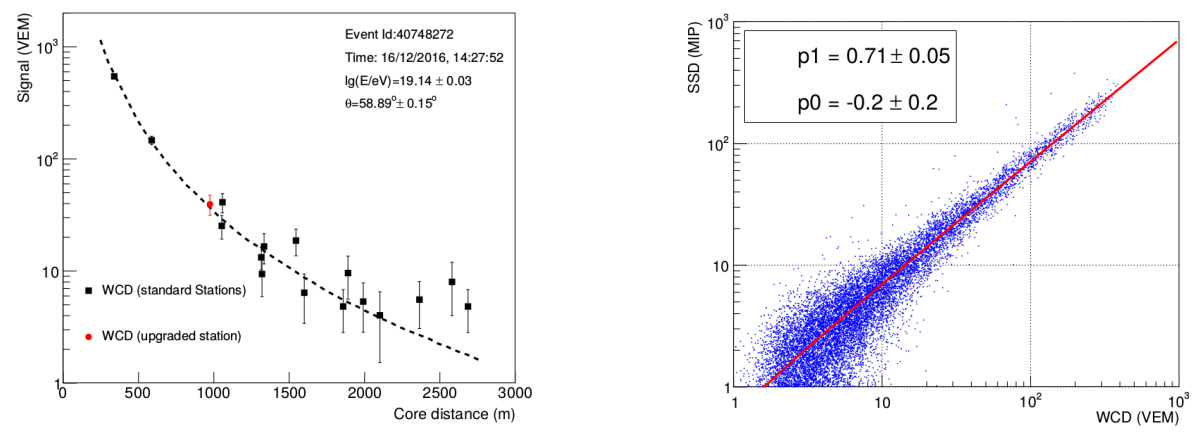

Figure 8. Left: one event reconstructed with the $1500 \mathrm{~m}$ array in close proximity to the EA. The reconstructed signals in the EA are compared with the lateral distribution function of the event; Right: correlation of the signals of the SSD and the WCD [15].

An SSD module consists in two $3.8 \mathrm{~m} \times 1.3 \mathrm{~m}$ sub-modules, each composed of 24 bars of extruded scintillator. The scintillation light produced in the bars is collected by optical fibers and read by a photomultiplier [15]. The layout of a SSD module is shown in Figure 7-Left. The first twelve stations of AugerPrime, forming the Engineering Array of the upgrade, were deployed at the Pierre Auger Observatory in September 2016 and have been in continuous data taking mode since begin of October 2016 producing signals that are in good agreement with expectations as shown in Figure 8.

To verify and fine-tune the methods used to extract shower muon content using the SSD and WCD stations, an underground muon detector (AMIGA) will be installed to provide a direct measurement of the muon content of a sample of showers observed by the upgraded Auger SD. The AMIGA detector consists of 61 units deployed on a $750 \mathrm{~m}$ grid, instrumenting a total area of $23.5 \mathrm{~km}^{2}$. Each units consists of a plane of plastic scintillator of about $30 \mathrm{~m}^{2}$ that will be buried about 2.3 meters underground. The start of the deployment of the upgraded stations is expected in January 2018 and will last about two years.

\section{Conclusions}

After more than ten year of activity the Pierre Auger Observatory reached important milestones in UHECRs research field, but a fully consistent interpretation of results is limited by the lack of knowledge at the highest energies. The suppresion of the flux at the highest energies was observed without any doubt but, so far, its origin is unclear. The search for photons and neutrinos constrain models based on proton-dominated sources and the study of the distribution of the air-showers $X_{\max }$ suggests a mixed chemical composition of cosmic rays at the highest energies. The analysis of the distribution of the arrival directions of UHECRs demonstrates the extra-galactic origin of these particles and indicates possible correlations with astrophysical sources.

Thanks to the extension of the data taking up to 2024 the number of collected events will be doubled in comparison to the statistics collected up to now, with the advantage that every future event will have mass information and will allow us to better address some of the most pressing questions in UHECRs physics. 


\section{References}

[1] The Pierre Auger Collaboration, Nucl. Instrum. Meth. A 798, 172 (2015)

[2] F. Fenu for the Pierre Auger Collaboration, Proc. of the $35^{\text {th }}$ ICRC, Busan, (2017) arXiv:1708.06592v2

[3] J. Hersil et al., Phys. Rev. Lett. 6, 22 (1961)

[4] J. Bellido for the Pierre Auger Collaboration, Proc. of the $35^{\text {th }}$ ICRC, Busan, (2017) arXiv:1708.06592v2

[5] The Pierre Auger Collaboration, Phys. Rev. D 90, 122005 (2014)

[6] The Pierre Auger Collaboration, Phys. Rev. D 90, 122006 (2014)

[7] The Pierre Auger Collaboration, Science 57, 1266-1270 (2017)

[8] U. Giaccari for the Pierre Auger Collaboration, Proc. of the $35^{\text {th }}$ ICRC, Busan, (2017) arXiv:1708.06592v2

[9] M. Niechciol for the Pierre Auger Collaboration, Proc. of the $35^{\text {th }}$ ICRC, Busan, (2017) arXiv:1708.06592v2

[10] E. Zas for the Pierre Auger Collaboration, Proc. of the $35^{\text {th }}$ ICRC, Busan, (2017) arXiv:1708.06592v2

[11] The Pierre Auger Collaboration, Phys. Rev. D 91, 032003 (2015)

[12] The Pierre Auger Collaboration, Phys. Rev. Lett. 117, 192001 (2016)

[13] The Pierre Auger Collaboration, The Pierre Auger Observatory Upgrade AugerPrime: Preliminary Design Report, (2016) arXiv:1604.03637

[14] A. Castellina for the Pierre Auger Collaboration, Proc. of the $35^{\text {th }}$ ICRC, Busan, (2017) arXiv:1708.06592v2

[15] D. Martello for the Pierre Auger Collaboration, Proc. of the $35^{\text {th }}$ ICRC, Busan, (2017) arXiv:1708.06592v2 